\title{
ON THE SET OF SOLVABLE $n$-PERSON GAMES
}

\author{
BY BEZALEL PELEG
}

Communicated by A. W. Tucker, July 20, 1959

The set $G_{n}$ of all $n$-person games ${ }^{1}$ may be considered a convex subset of a euclidean space; $G_{n}$ has dimension $2^{n}-n-2$. Although it has not been proved that all games possess a solution, large classes of solvable games have been discovered. In [2], Shapley defined a certain class of solvable games-the quota games-and showed that from the point of view of dimension, the set $Q_{n}$ of all $n$-person quota games constitutes a considerable part of $G_{n}$. For $n$ odd (the more favorable case), the dimension of $Q_{n}$ differs from that of $G_{n}$ by $(n-1)(n-2) / 2$. Shapley also showed that by suitable extensions, it is possible to increase slightly the dimensionality of the set of games known to have a solution. Here we introduce a modification of the notion of quota called a partial quota, and demonstrate the existence of partial quota solutions for all partial quota games (games possessing a partial quota). We then show that the set of all partial quota games has dimension equal to that of $G_{n}$. Thus the probability is positive that an $n$-person game "picked at random" has a solution. ${ }^{2}$ A similar result holds for zero-sum games: the set of zero-sum partial quota $n$-person games has dimension equal to that of all zero-sum $n$-person games.

Notation will be as in $[2]$, except that $(0,1)$ normalization will be used throughout. We will show that a sufficient condition for a game $v$ to possess a partial quota solution is that there is at least one triple $\{i, j, k\}$ of players such that

$$
v_{i j}+v_{i k}+v_{j k} \geqq 2 .
$$

This single inequality does not imply any equalities involving the values of the characteristic function; hence the set of games satisfying (1) is of the same dimension as $G_{n}$.

A brief sketch of the proof is as follows: Let $T$ be a subset of $I$ with at least two members. A partial quota for $T$ is a vector $\left(\rho_{i}\right)_{i \in T}$ satisfying

$$
\text { for all } i, j \in T, \quad \rho_{i}+\rho_{j}=v_{i j}
$$

\footnotetext{
${ }^{1}$ Cooperative games with side payments, as defined by their characteristic functions [1]. We do not distinguish between $S$-equivalent games.

${ }^{2}$ Gillies [5] has recently obtained a similar result (i.e. that a positive fraction of all games is solvable). His methods are completely different from ours, and lead to a different set of solvable games.
} 
and

$$
\sum_{j \in T} \rho_{j} \geqq 1
$$

Suppose a game $v$ has a partial quota for a subset $T$ of $I$. If $T=I$, then $v$ is known to be solvable [4]. If $T \neq 1$, let $p$ be a player not in $T$. Define a discriminatory quota $\left(\omega_{i}\right)_{i \in I}$ by

$$
\omega_{i}=\left\{\begin{array}{lr}
\rho_{i} & i \in T, \\
1-\sum_{j \in T} \rho_{j} & i=p, \\
0 & \text { otherwise. }
\end{array}\right.
$$

It can be shown that there is at most one weak player, i.e. at most one player for whom $\omega_{i}<0$. Let $i \rightarrow b_{i}$ be an arbitrary function from $T$ into itself, such that for each $i$ in $T, b_{i} \neq i$. If there is no weak player, then

$$
V_{b}=\bigcup_{i \in T}\left[\omega, \gamma^{i b_{i}}\right]
$$

is a solution of the game $v$. If there is a weak player, let $o$ denote the weak player; then

$$
V_{b}=\bigcup_{i \in T-\{o\}}\left[\gamma^{o i}, \gamma^{i o b_{i}}\right]
$$

is a solution of $v$. The proofs are quite similar to Shapley's proofs.

Let $T$ be an arbitrary subset of $I$ with exactly 3 members. Then the equations (2) always have a unique solution. Hence to show that $v$ has a partial quota for $T$, it is sufficient to show that (3) holds; but (3) is in this case equivalent to (1). This completes our proof.

Returning to $T$ general, we give the interpretation of the partial quota solution for $T \neq I$ when there is no weak player. Each member of $T$ chooses a beneficiary in $T$. The standard of behavior gives every member of $T$ his quota, except possibly that one member of $T$ may accept less than his quota and give the difference to his beneficiary. On the other hand, the standard of behavior viciously discriminates against players not in $T$; each of these receives only his personal minimum, namely 0 . When there is a weak player, or when $T=I$, the interpretation is slightly more complicated but not essentially different.

Note that when $T$ has an odd number of members, it may very well be that $v(I-T)>0$. In this case the players not in $T$ can improve their payoff without needing any help from within $T$, so it is rather curious that they can be effectively "discriminated" against. Another 
interpretation is that $T$ exacts "tribute" from $I-T$, in a manner somewhat analogous to that described in [1] in connection with decomposable games.

The result that the dimension of the set of solvable games equals that of $G_{n}$ can be obtained without appealing to quota considerations, by considerations of the "extended" theory introduced in Chapter IX of [1] (Composition and Decomposition of Games). Although the extended theory is developed in [1] for zero-sum games only, the parts that we use can be shown to be valid for general-sum games as well. Let $T$ be an arbitrary subset of $I$. Then it can be shown that a necessary and sufficient condition that a solution of $v \mid T$ in $E(1-v(T))$ be inflatable ${ }^{3}$ to an ordinary solution of $v$ is that

$$
1-v(T) \leqq|\Gamma|_{2}
$$

where the $|\Gamma|_{2}$ is that of $v \mid T$ (of course $v \mid T$ is no longer $(0,1)$ normalized). Now let $T=\{i, j, k\}$; then it can be shown that (1) implies (4). It remains only to solve $v \mid T$ in $E(1-v(T))$; to this end, define an auxiliary game $v^{\prime}$ on $T$ by $v^{\prime}(T)=1, v^{\prime}(S)=v(S)$ for all proper subsets $S$ of $T$. Then ordinary solutions to $v^{\prime}$ are solutions to $v \mid T$ in $E\left(1-v(T)\right.$ ) (and conversely). But since $v^{\prime}$ is a (general-sum) threeperson game, it is solvable [1, pp. 573-581], and our proof is complete.

It is rather interesting that the result we obtain by the general method just described is "hardly" better than that obtained by the very special quota method. More precisely, the set of all games satisfying (4) when $T=\{i, j, k\}$ is greater than the set of all games satisfying (1) only by a set of much lower dimension. ${ }^{4}$ Thus if we neglect sets of lower dimension, both methods lead (for $T=\{i, j, k\}$ ) to (1). When $T$ has more than three players, the partial quota method leads to interesting solutions, but the games solvable by this method form a set of much lower dimension. ${ }^{5}$ The methods of the extended theory, on the other hand, might lead to a significant widening of the set of games known to be solvable, but presumably not before further work has been done on the general-sum four-person game (and possibly higher games).

I wish to thank Dr. R. Aumann for several helpful discussions.

${ }^{3}$ The inflation of $[3$, p. 335$]$ is meant; that is, constant amounts are assigned to the players in $I-T$ (in our case, they must be 0 ).

4 (4) implies (1) except possibly when $v(T)=1$; the dimension of the set of all $n$-person games for which $v(T)=1$ is lower than the dimension of $G_{n}$ by $2^{n-2}-n+1$.

5 Though still a good deal higher than that of $Q_{n}$. 


\section{REFERENCES}

1. J. von Neumann and $\mathrm{O}$. Morgenstern, Theory of games and economic behavior, Princeton University Press, 1944, 2d ed., 1947.

2. L. S. Shapley, Quota solutions of $n$-person games, Contributions to the Theory of Games II, Princeton University Press, 1953, pp. 343-360.

3. D. B. Gillies, Discriminatory and bargaining solutions to a class of symmetric $n$-person games, Contributions to the Theory of Games II, Princeton University Press, 1953, pp. 325-342.

4. G. K. Kalisch, Generalized quota games, Report of an Informal Conference on the Theory of $n$-Person Games, Princeton University, 1953, pp. 1-3.

5. D. B. Gillies, Solutions to general non-zero-sum games, Contributions to the Theory of Games IV, Princeton University Press, 1959, pp. 47-86.

The Hebrew University, Jerusalem, Israel 
